Hughes W. H. (1955). J. gen. Microbiol. 12, 265-268

\title{
The Inheritance of Differences in Growth Rate in Escherichia coli
}

\author{
By W. HOWARD HUGHES \\ Wright-Fleming Institute, St Mary's Hospital Medical School, \\ London, W.2
}

SUMMARY: In cultures from single cells of Escherichia coli grown under anaerobic conditions there are variations in rate of growth of the individual colonies as measured by their diameter after a short incubation. When small and large colonies are selected the populations derived from them inherit the tendency to produce correspondingly smaller or larger colonies than the mean of those produced by the parent strain. When the growth conditions are altered then the selected character will be lost.

It was noticed that in Escherichia coli, strain Brent, the colonies grew at different rates under anaerobic conditions in a de Fonbrune oil chamber. This was a character that could be studied in much the same way as size in higher plants (cf. East, 1915) and it seemed possible that fast- and slow-growing strains could be selected and that it might be shown that the rate of growth was an inherited characteristic.

At first accurate histograms were not obtained since the random distribution of an implant on a solid medium results in some of the colonies being derived from more than one parent cell. Also, for the test to be convincing the parent strain and the various daughter strains selected from it should be cultivated on the same batch of medium under identical conditions. Fortunately a technique has been found which enables as many as $\mathbf{5 0 0}$ single cells from five strains to be dealt with in the same oil chamber on samples of the same medium.

\section{METHOD}

A single cell was picked by means of the micromanipulator pipette and transferred to a drop of Hartley's digest broth where it was incubated for $\mathbf{3} \mathrm{hr}$. at $37^{\circ}$. The drop of suspension was withdrawn from the oil chamber and transferred into $10 \mathrm{ml}$. of broth and there incubated for a further $3 \mathrm{hr}$. at $37^{\circ}$ with continuous agitation. This suspension was retained and stored at $4^{\circ}$ and formed the parent strain, suspension no. 1.

Solid medium was prepared from the same broth with $2 \%$ Kobe no. 1 shred agar. A layer of the melted agar was poured on a sterilized glass slide; after cooling, the agar was trimmed and cut up into squares $0.5 \mathrm{~cm}$. across and one of these was transferred to the coverslip of an oil-chamber with a scalpel. A loopful of the stored culture was spread on this square, the coverslip was inverted and the chamber filled and incubated at $37^{\circ}$ for $3 \mathrm{hr}$.

After incubation the colonies were inspected and the smallest was picked into a drop of broth. A segment of one of the largest colonies, containing 
about the same number of cells, was similarly treated. These two new suspensions, the small no. 2 and the large no. 3, were transferred, without incubation in the chamber, into tubes of broth, incubated for $3 \mathrm{hr}$. at $37^{\circ}$ and then stored with the parent suspension at $4^{\circ}$. In turn, samples of these two suspensions were spread on agar blocks and incubated and the smallest colony from no. 2 and a segment of the largest from no. 3 taken to form another pair, no. 4 and no. 5 respectively.

On the following day the three stored cultures were taken and $0.1 \mathrm{ml}$. of each was incubated with $9 \mathrm{ml}$. of broth for $2 \mathrm{hr}$. at $37^{\circ}$. The oil chamber was prepared with five blocks of agar arranged so that they were equidistant from the edge of the coverslip. The distance from the edge of the coverslip is important since, when the blocks were not separated from the air by a margin of $0.3 \mathrm{~mm}$. or more, it was found that colonies developing at the exposed edge had an advantage and uniform results were not obtained.

A loopful of suspension no. 1 was now spread on the first block; similarly, suspensions nos. 2 and 3 were given a block each. Suspensions nos. 4 and 5 had been retained in the oil chamber at $4^{\circ}$ and as much as possible of each of these was transferred to the appropriate remaining block without further subculture, the coverslip was inverted and the oil chamber filled.

While it is difficult by micromanipulator to plant out large numbers of single cells, it proved relatively easy to thin out the implant (made with a loop in the ordinary way) by removing the excess cells with a micropipette so that there was not more than one cell to each field of the microscope at a magnification of $\mathbf{2 4 0 .}$

The manipulation took place at room temperature and it was possible to obtain the necessary 100 single cells on each of the five blocks before their first division had been completed. When 100 cells had been so isolated on one block the area was marked and any remaining cells left unthinned.

The preparation was now incubated at $37^{\circ}$ for $3 \mathrm{hr}$., cooled in the refrigerator to stop further growth and the colony diameters measured using an eyepiece micrometer. The results are expressed in Fig. 1 as direct measurements of colony diameter.

\section{RESULTS}

The histograms of the subcultures show that from the single cell giving the first culture it is possible to select out populations which grow either more or less rapidly than the parent strain in the particular environment provided. There was no detectable delay in growth starting in the slow-growing culture. In culture 4 the small colony strain gave rise to cells which were unable to divide and many of which gave the long forms previously reported with this organism (Hughes, 1953).

Once small colonies have been obtained, with re-selection a strain can be kept which produces uniformly small colonies under the conditions in the oil chamber. When this strain was grown aerobically in broth the turbidity reached was not obviously different from that of the parent or the large colony strains. When, however, it was returned to the oil chamber it showed the small 
colony character. Three or more overnight subcultures in aerobic broth without re-selection, however, were followed by a blurring of the pattern and the strains approximated progressively to the parent.

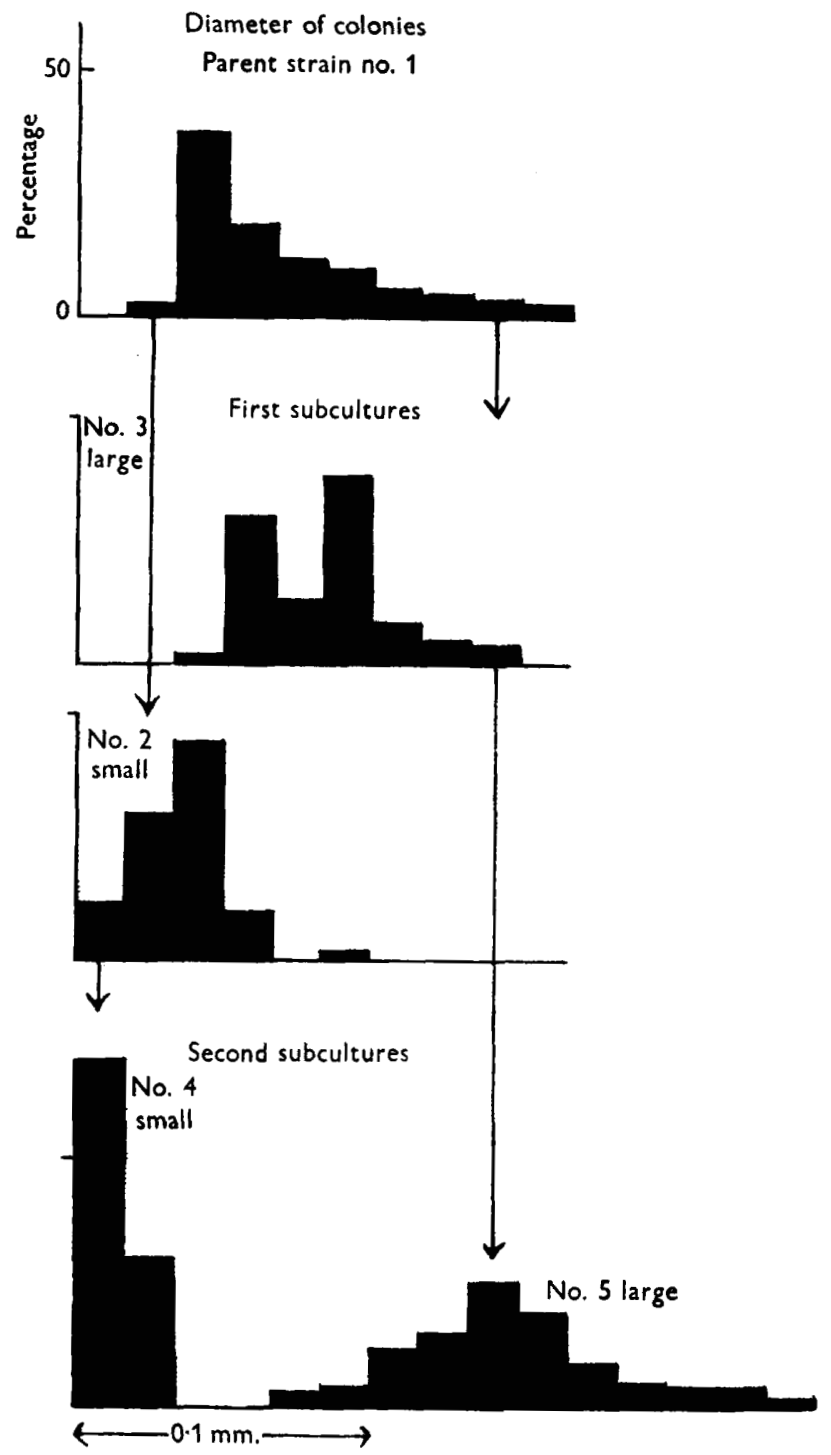

Fig. 1. Histogram showing rates of growth of colonies from single cells of Escherichia coli grown under anaerobic conditions. Measurements of colony diameter made after $3 \mathrm{hr}$. incubation at $37^{\circ}$. Re-selection of the subcultures indicated by arrows.

\section{DISCUSSION}

It would appear that variation takes place in cultures of organisms derived from a single cell. This variation is apparently not due to minor differences in the environment since in these experiments such differences were reduced to 
a minimum. The populations breed true for just as long as no other selection by a different culture medium or atmospheric condition is applied to them. It is not possible at this stage to indicate what it is in the large colony strain that gives it an advantage over the small. Both seem to grow equally well in ordinary aerobic culture.

The general results agree well with those obtained by Rogers (1953). When he was selecting strains for hyaluronidase production, he also used an entire population but felt that in this there was a relatively small number of discrete populations differing in their ability to form enzyme. Here it is not obvious that any sharp lines can be drawn between the various populations, though further experience may show that the differences between them can be broken down into discrete steps.

\section{REFERENCES}

EAST, E. M. (1915). Studies in size inheritance in Nicotiana. Genetics, 1, 164.

Hughes, W. H. (1953). The origin of the L-form variants in anaerobic cultures of Bacterium coli. J. gen. Microbiol. 8, 307.

Rogers, H. J. (1953). Variant populations within a hyaluronidase-producing culture of Staphylococcus aureus. J. Path. Bact. 66, 545.

(Received 11 October 1954) 